Revue internationale P.M.E.

Économie et gestion de la petite et moyenne entreprise

\title{
De la nécessité de poursuivre la recherche sur la planification des PME
}

\section{Hervé Goy}

Volume 14, numéro 2, 2001

URI : https://id.erudit.org/iderudit/1008693ar

DOI : https://doi.org/10.7202/1008693ar

Aller au sommaire du numéro

\section{Éditeur(s)}

Presses de l’Université du Québec

ISSN

0776-5436 (imprimé)

1918-9699 (numérique)

Découvrir la revue

Citer cette note

Goy, H. (2001). De la nécessité de poursuivre la recherche sur la planification des PME. Revue internationale P.M.E., 14(2), 85-108.

https://doi.org/10.7202/1008693ar

\section{Résumé de l'article}

Cet article suggère que l'état actuel de la recherche sur la planification des PME ne permet pas de contribuer à la nécessaire théorisation du phénomène. Ainsi, de nombreux chercheurs se sont intéressés à la valeur de la planification en PME. Ces travaux ont donné lieu à des résultats très hétérogènes, voire contradictoires, sur l'existence d'une relation statistique entre la présence de la planification et divers indicateurs de performance, le plus souvent financière (et parfois organisationnelle), de l'organisation. Par ailleurs, certains aspects de la recherche sur la planification des PME restent flous. En effet, deux catégories de recherches restent à un niveau insatisfaisant de connaissance sur ce thème. Le premier groupe de travaux, que l'on peut qualifier de descriptif, concerne l'étude des pratiques de planification des PME. Les recherches concernées ne permettent pas, en effet, de saisir la réalité des pratiques de planification dans ces firmes, du fait notamment d'une définition de la planification a priori, mais aussi du choix d'une méthodologie quantitative, inefficace selon nous, pour caractériser le comportement planificateur des PME. La seconde catégorie d'études, de nature normative, traite de la question des processus de planification de ces entreprises. L'ensemble de ces contributions constitue un apport d'une richesse intéressante, malgré leur variété rendant l'effort de synthèse difficile. Pourtant, il reste possible de dresser le constat de l'absence de consensus dans la littérature sur l'élaboration du plan stratégique, ainsi que du manque d'une méthode d'adaptation d'un modèle quelconque aux exigences d'une PME.

Ainsi, dans un objectif de contribution à l'effort de théorisation de la planification en PME, nous proposons un cheminement de recherche différent, caractérisé par une perspective inductive. De cette manière, il devrait être possible de mettre en évidence une forme de modélisation de la planification dans les entreprises de petite et moyenne dimension.
Ce document est protégé par la loi sur le droit d'auteur. L'utilisation des services d'Érudit (y compris la reproduction) est assujettie à sa politique d'utilisation que vous pouvez consulter en ligne.

https://apropos.erudit.org/fr/usagers/politique-dutilisation/ 


\section{Notes de recherche De la nécessité de poursuivre la recherche sur la planification des PME}

Hervé GOY

Centre d'études et de recherches appliquées à la gestion (CERAG)

de l'Université de Grenoble II

\section{MOTS CLÉS}

\section{Stratégie - Planification - PME - Méthodes qualitatives Grounded Theory}

\section{RÉSUMÉ}

Cet article suggère que l'état actuel de la recherche sur la planification des PME ne permet pas de contribuer à la nécessaire théorisation du phénomène. Ainsi, de nombreux chercheurs se sont intéressés à la valeur de la planification en PME. Ces travaux ont donné lieu à des résultats très hétérogènes, voire contradictoires, sur l'existence d'une relation statistique entre la présence de la planification et divers indicateurs de performance, le plus souvent financière (et parfois organisationnelle), de l'organisation. Par ailleurs, certains aspects de la recherche sur la planification des PME restent flous. En effet, deux catégories de recherches restent à un niveau insatisfaisant de connaissance sur ce thème. Le premier groupe de travaux, que l'on peut qualifier de descriptif, concerne l'étude des pratiques de planification des

\section{L'AUTEUR}

HERVÉ Gor détient un doctorat en sciences de gestion. Ses principaux domaines d'intérêt sont la stratégie et l'organisation des petites et moyennes entreprises. Présentement attaché temporaire d'enseignement et de recherche à l'IUT GACO de Saint-Étienne, il intervient par ailleurs dans les formations de l'École supérieure des affaires et de l'École supérieure de commerce de Grenoble. Adresse : IUT département GACO, 28, avenue Léon-Jouhaux, 42023 SaintÉtienne, cedex 2, France. Courriel : <hervegoy@hotmail.com> 
PME. Les recherches concernées ne permettent pas, en effet, de saisir la réalité des pratiques de planification dans ces firmes, du fait notamment d'une définition de la planification a priori, mais aussi du choix d'une méthodologie quantitative, inefficace selon nous, pour caractériser le comportement planificateur des PME. La seconde catégorie d'études, de nature normative, traite de la question des processus de planification de ces entreprises. L'ensemble de ces contributions constitue un apport d'une richesse intéressante, malgré leur variété rendant l'effort de synthèse difficile. Pourtant, il reste possible de dresser le constat de l'absence de consensus dans la littérature sur l'élaboration du plan stratégique, ainsi que du manque d'une méthode d'adaptation d'un modèle quelconque aux exigences d'une PME.

Ainsi, dans un objectif de contribution à l'effort de théorisation de la planification en PME, nous proposons un cheminement de recherche différent, caractérisé par une perspective inductive. De cette manière, il devrait être possible de mettre en évidence une forme de modélisation de la planification dans les entreprises de petite et moyenne dimension.

\begin{abstract}
This article suggests that the current state of research, regarding planning in small and medium-sized businesses, does not contribute to the necessary theorization of such activity. So, several studies have been handled about the value of planning for small business. The outcome was either heterogeneous or contradictory results on the potential correlation between planning and financial (or even organizational) performance results. On the other side, some aspects of the planning study remains wooly. Actually, two research categories stay at an unsatisfactory level of knowledge on this subject. The first one, more descriptive, focuses on the study of current practices. Researches from this angle do not capture the reality of planning activity, mainly because of the a-priori assumption of such planning, but also due to the quantitative methodology used to describe the planning approach. The second one, normative in essence, deals with planning processes. Those various contributions bring some enriching learning but their diversity does not ease synthesis. However, it is possible to highlight the lack of consensus in the documentation on planning and the missing evolution of planning methodology to comply with small and mediumsized companies requirements.
\end{abstract}

Therefore, in order to contribute to the on-going theorization of planning in small business, we propose a different approach, distinguished by an inductive perspective. In this way, it should be possible to identify a new model for planning concerning small and medium-sized businesses.

\title{
RESUMEN
}

Este artículo sugere que el estado actual de la investigación sobre la planificación de las PyMEs no permite contribuir a la teorización del fenómeno. Así, numeros investigadores se han interesado a la valor de la planificación de las PyMEs. Estes trabajos dan resultados muy heterogeneos incluso contradictorios sobre la existencía de una relación estatistica entre la presencía de la planificación y varios indicatores del rendimiento, la mayoria de las veces financiero (y unas veces organizacional) de la empresa. Ademas, unos aspectos de la investigación sobre

Revue internationale P.M.E., vol. 14, nº 2, 2001

(C) 2001 - Presses de l'Université du Québec

Édifice Le Delta I, 2875, boul. Laurier, bureau 450, Sainte-Foy, Québec G1V 2M2 • Tél. : (418) 657-4399 - www.puq.uquebec.ca

Tiré de : Revue internationale P.M.E., vol. $14, \mathrm{n}^{\circ} 2$, sous la direction de Pierre-André Julien. 
la planificación de las PyMEs quedan vagos. En efecto, dos categorías de investigaciones quedan a un nivel insatisfactorio de conocimiento sobre este tema. El primero grupo de estos trabajos, que se llama descriptivo, concierne el estudio de las practícas de la planificación en las PyMEs. Estas investigaciones no permiten entender la realidad de la planificación en estas firmas, porque utilizan una definición de la planificación a priori, y tambien porque han echo la elección de una metodologia quantitativa, ineficaz sola para caracterizar el comportamiento de planificación de las PyMEs. La segunda categoría de estudios, de natura normativa, trate del problemo de los procesos de planificación de estas empresas. Juntas, estas contribuciones constituyen un aportación rico, pero, a causa de su variedad, una síntesis queda dificil. No obstante, queda posible comprobar la absencía de consensus en la literatura sobre la elaboración del plano estratégico, asi que la falta de un método de adaptación de un cualquiera modelo a las exigencias de las PyMEs.

Asi, para contribuir al esfuerzo de teorización de la planificación en las PyMEs, propuestemos un camino de investigación diferente, caracterizado por una perspectiva inductiva. De este manera, debera estar posible poner de manifesto una forma de modelisación de la planificación en las empresas de pequeña et media dimensión.

\section{ZUSAMMENFASSUNG}

Dieser Artikel legt nahe, dass der aktuelle Forschungsstand über die Planung der KMU es nicht erlaubt, die Notwendigkeit einer theoretischen Betrachtung abzulehnen. Umso mehr sind eine Vielzahl von Forschern interessiert am Stellenwert der Planung in KMU. Diese Arbeiten haben sehr heterogene Resultate aufgezeigt, sogar widersprüchliche. Einerseits was die Existenz einer statistischen Beziehung zwischen der Präsenz der Planung und verschiedenen Leistungsindikatoren, vor allem finanziellen (und manchmal organisatorischen) Indikatoren der Organisation betrifft. Andererseits bleiben verschiedene Aspekte der Forschung zur Planung in KMU unklar. In Wirklichkeit bleiben zu diesem Thema zwei Forschungskategorien auf einem unbefriedigendem Wissensniveau. Die erste Gruppe von Arbeiten, die man als beschreibende einstufen kann, betrifft die Untersuchung von Planungspraktiken in KMU. Die betroffenen Untersuchugen erlauben in Wirklichkeit nicht, die Realität der Planungspraxis in diesen Unternehmen zu erfassen, sei es eine a priori Definition der Planung zu geben, aber auch die Wahl der quantitativen Methodologie ist nach uns uneffizient, das Planungsverhalten der KMU zu charakterisieren. Die zweite Kategorie der Studie behandelt die Frage der Planungspropezesse von Unternehmen. Trotz ihrer Vielfalt bedeutet das Gesamte dieser Beiträge ein interessanter und reicher Beitrag. Es ist aber festzuhalten, dass in der Literatur keine Übereinstimmung besteht zur Ausarbeitung des strategischen Planes sowie keine Modell- Methode besteht für KMU.

Mit dem Ziel ein Beitrag zu leinsten zur theoretischen Betrachtung der Planung in KMU schlagen wir einen anderen Forschungsweg vor, der charakterisiert ist durch eine induktive Perspektive. Durch diese Art sollte es möglich sein die Planung von kleinen une mittleren Undernehmen zu modellieren.

Revue internationale P.M.E., vol. 14, n 2, 2001

(C) 2001 - Presses de l'Université du Québec

Édifice Le Delta I, 2875, boul. Laurier, bureau 450, Sainte-Foy, Québec G1V 2M2 • Tél. : (418) 657-4399 - www.puq.uquebec.ca

Tiré de : Revue internationale P.M.E., vol. 14, $\mathrm{n}^{\circ} 2$, sous la direction de Pierre-André Julien. 


\section{Introduction}

Depuis le début des années 1980, la recherche sur les stratégies de planification des entreprises de petite et moyenne dimension se structure principalement autour d'études empiriques, sous l'impulsion d'auteurs comme Robinson et Pearce (1984), Bracker et Pearson (1986), ou bien encore Ackelsberg et Arlow (1985) pour ne citer qu'eux. Les apports de ces travaux sont considérables, mais, dans le même temps, appréhendés de manière globale, nous pouvons leur reprocher notamment leur manque de continuité d'une étude à l'autre ainsi que l'impossibilité d'établir des comparaisons entre recherches du fait d'analyses et de secteurs différents, d'échantillons hétérogènes, etc. (d'Amboise et Bakanibona, 1990). Ainsi, le courant de recherches sur la planification des PME semble avoir échoué, jusqu'à présent, à contribuer à faire émerger une théorie de la planification dans les firmes de petite et moyenne dimension. Cet échec, en plus des raisons évoquées ci-dessus par d'Amboise et Bakanibona, est attribuable à plusieurs causes. L'hétérogénéité des définitions données à la PME, le faible intérêt porté au contenu des plans et à leur mise en œuvre et le fait de retenir comme seul interlocuteur des études le dirigeant de la firme sont autant d'explications possibles au manque de cohésion du champ de recherche sur la planification en PME. Mais sans doute l'utilisation de méthodologies exclusivement quantitatives, comme le précise Orpen (1985), contribue-t-elle également à aboutir à ce que nous considérons être un état insatisfaisant de la recherche en stratégie sur la planification des PME.

La notion de planification, telle qu'elle a été développée en management stratégique sous des appellations diverses (PPBS, planification à long terme ou planification stratégique) est principalement issue de grands cabinets-conseils, comme le Boston Consulting Group. Ceux-ci ont contribué au développement, dans les grandes firmes américaines à l'origine, d'outils et de techniques susceptibles de les aider à « définir la mission de leur entreprise, à déterminer leurs objectifs à long terme et à choisir les stratégies pour les atteindre » (Steiner, 1979). Dès lors, certains chercheurs ont tenté de donner au terme de planification une définition appropriée à la PME. Il s'agit ainsi pour Golde (1964) d'un «processus qui s'interroge de manière systématique et globale sur le futur de l'entreprise ». Montebello, Saias et Greffeuille (1974) intègrent, pour leur part, les notions d'adaptation et d'anticipation dans la définition qu'ils donnent de la planification en PME. Pour Bamberger (1979), la planification dans les firmes de petite et moyenne dimension apparaît comme un concept stratégique qui relève à la fois de la créativité et du contrôle. Plus récemment, Calori, Véry et Arrègle (1997) considèrent la planification stratégique en PME comme la combinaison de l'anticipation et de la formalisation. Pour l'ensemble de ces auteurs, et pour d'autres encore, la planification en PME s'apparente donc à un processus global, systématique, continu et analytique, sans pour autant qu'il y ait consensus sur la définition à donner au phénomène 
observé. Or, ces caractéristiques nous paraissent largement inspirées de l'analyse de la planification en grande entreprise et, de fait, nous semblent susceptibles d'être remises en cause en ce qui a trait à la PME. En effet, intuitivement, ces dimensions ne nous semblent pas refléter la réalité de la pratique planifiée de la stratégie dans les firmes de petite et moyenne dimension.

Pour toutes ces raisons, nous nous posons donc la question suivante : peut-on se satisfaire de l'état actuel de la recherche sur la planification dans les entreprises de petite et moyenne dimension? Signalons toutefois que l'objectif de cet article est moins de relever les limites des travaux existants (dont les apports ne peuvent être ignorés) que de proposer des voies de recherche différentes dans l'étude de la planification des PME.

Afin de répondre à cette interrogation, il nous semble indispensable de passer en revue les différentes contributions relatives à l'étude de la planification dans les PME, selon deux principaux axes inspirés de la synthèse proposée par Robinson et Pearce (1984) dans leur étude remarquable sur la question. C'est pourquoi nous nous intéresserons, tout d'abord, aux apports des travaux sur l'étude de la valeur de la planification dans les PME qui constituent l'essentiel de la littérature sur ce thème. Nous constaterons ainsi, à travers l'analyse de la relation de la planification avec quelques indicateurs de performance de la firme, que ces recherches aboutissent dans l'ensemble à des conclusions diverses. Ce survol nous permettra par la suite d'évoquer, dans une seconde partie, les questions fondamentales auxquelles ces études, selon nous, ont du mal à répondre. Ainsi, il conviendra de nous intéresser aux développements de nature descriptive, concernant les pratiques des PME en matière de planification, ainsi qu'aux apports de travaux résolument normatifs, sur «le» processus à mener dans ce type d'entreprise. Finalement, nous soumettrons en fin d'article une perspective de recherche sur l'étude de la planification en PME, qui nous semble mobilisable dans le nécessaire effort de conceptualisation qui reste à produire sur la question.

\section{La recherche sur la planification en PME : un courant principalement fondé sur l'analyse de la valeur}

Si la question de l'utilité de la planification en PME, sur laquelle nous ne souhaitons pas épiloguer ici, reste ouverte au débat (Green, 1982; Montebello, Saias et Greffeuille, 1974 ; Scarborough et Zimmerer, 1987 ; Baker, Addams et Davis, 1993 ; Unni, 1981), il en est une autre qui ne cesse de faire couler de l'encre depuis quelques années maintenant : celle de la relation entre planification et performance dans les petites et moyennes entreprises.

Revue internationale P.M.E., vol. 14, nº 2, 2001

(C) 2001 - Presses de l'Université du Québec

Édifice Le Delta I, 2875, boul. Laurier, bureau 450, Sainte-Foy, Québec G1V 2M2 • Tél. : (418) 657-4399 - www.puq.uquebec.ca

Tiré de : Revue internationale P.M.E., vol. $14, \mathrm{n}^{\circ} 2$, sous la direction de Pierre-André Julien. 


\subsection{L'étude du lien entre planification et performance des PME : des résultats contradictoires}

Dans l'esprit de Robinson et Pearce (1984), d'Amboise et Bakanibona (1990) ont réalisé une synthèse de travaux empiriques relatifs à l'étude de la planification dans les PME, mettant en avant les grands axes suivants :

- un lien statistiquement positif entre l'existence de la planification et la performance des PME (Ackelsberg et Arlow, 1985 ; Sexton et Van Auken, 1985);

- un lien positif entre le degré de formalisation de la planification stratégique et la performance des PME pour Orpen (1985), mais négatif pour Robinson et Pearce (1983) et Ackelsberg et Arlow (1985);

- un lien positif entre la sophistication de la planification stratégique et la performance des PME pour Bracker et Pearson (1986) et Bracker, Keats et Pearson (1988), entre l'intensité de la planification et la performance pour Robinson et al. (1984), entre la structuration de la planification stratégique et la performance pour Bracker, Keats et Pearson (1988) et entre la qualité de la planification et la performance en PME pour Orpen (1985);

- concernant plus particulièrement l'étude de l'effet modérateur des variables de contingence, Bracker et Pearson (1986) trouvent un lien significatif entre l'expérience en planification et la performance, Robinson et al. (1984) ne notent aucune influence du stade de développement sur la relation planification-performance, Bracker, Keats et Pearson (1988) ne relèvent pas d'effet significatif de la taille de l'entreprise sur le lien planification-performance et émettent une forte présomption sur l'influence du type d'entrepreneur (opportuniste en l'occurrence) sur le degré de sophistication de la planification stratégique et la performance;

- un lien positif entre la planification opérationnelle et la performance, même en l'absence de planification stratégique pour Robinson, Logan et Salem (1986) ;

- enfin, pour Bracker et Pearson (1986) et, à la différence de Robinson (1982), il n'y a pas de lien entre l'utilisation de consultants et la performance des PME.

Plus récemment, Schwenk et Shrader (1993) ont analysé 14 études portant sur la planification stratégique formelle et la performance des PME et sont parvenus à la conclusion que la planification n'améliore pas nécessairement la performance et n'est pas seulement appropriée aux grandes entreprises. De même, à partir de leur étude quantitative auprès de $194 \mathrm{PME}$ du classement des 500 premières PME en forte croissance ( «INC. $500 »$ firms), Baker, Addams et Davis (1993) avancent les résultats suivants :

Revue internationale P.M.E., vol. 14, $\mathrm{n}^{\circ}$ 2, 2001 
- la contribution de la planification stratégique au rythme de croissance des firmes ;

- l'efficacité des plans dans l'accomplissement des buts planifiés;

- l'indétermination du sens du lien plans-profit, mais une association significative entre planification stratégique et profit des firmes.

En conclusion de cette étude, le couple planification stratégique formelle et plan d'activité écrit peut jouer un rôle significatif dans l'efficacité d'une PME.

Le tableau 1 récapitule les principales recherches traitant du lien planificationperformance. Malgré ces tentatives remarquables de synthèse de la multitude de travaux relatifs à l'étude de la performance des PME qui planifient, il n'en reste pas moins que la question n'est pas définitivement tranchée. Dès lors, certains chercheurs ont choisi de persévérer dans cette voie.

\subsection{Pour en finir avec la relation entre la planification et la performance en PME}

Pour Bracker et Pearson (1986), ces recherches diffèrent en raison de la faible taille des échantillons, de leur caractère ponctuel, de l'échec à intégrer les processus de planification constatés dans les modèles de management stratégique globaux, de l'ignorance du rôle du dirigeant dans le processus, de l'utilisation de tests statistiques inappropriés ou non robustes, de données non homogènes, de mesure inappropriée de la performance financière, de la non-distinction entre firmes jeunes et anciennes, ou bien encore de l'inattention portée à la taille de l'entreprise ainsi qu'à la nature statique ou dynamique de l'environnement. Soulevant le problème de la divergence des études sur le lien planification-performance des petites et moyennes entreprises, du fait de problèmes de mesure et de concept (reproductibilité difficile), Shrader, Mulford et Blackburn (1989) soulignent, pour leur part, la nécessité d'uniformiser la mesure de la planification, ce qui nous paraît difficile en l'absence de consensus sur ce qu'est la planification en PME!

Malgré ces constatations relatives à la difficulté d'harmoniser les recherches traitant du lien entre planification et performance en PME, certains auteurs ont proposé des angles d'étude nouveaux selon eux et susceptibles d'apporter une réponse claire à cette question. C'est ainsi que Schwenk et Shrader (1993) proposent de résoudre le problème des résultats contradictoires par l'utilisation d'une métaanalyse (transformation de l'information issue de tests statistiques dans chaque étude en une mesure commune) sur les recherches passées traitant du lien entre planification formelle et performance en PME. Ils estiment, en effet, qu'il y a un besoin crucial de conclure globalement sur la question du lien planificationperformance en PME. C'est ainsi qu' ils parviennent à la proposition selon laquelle 
TABLEAU 1

Recherches traitant du lien planification-performance en PME

\begin{tabular}{|c|c|c|c|c|c|}
\hline $\begin{array}{l}\text { Auteurs } \\
\text { et année }\end{array}$ & $\begin{array}{l}\text { Firmes } \\
\text { étudiées }\end{array}$ & $\begin{array}{l}\text { Secteur } \\
\text { d'activité }\end{array}$ & $\begin{array}{l}\text { Définition de la } \\
\text { PME retenue }\end{array}$ & $\begin{array}{l}\text { Méthodologie } \\
\text { employée }\end{array}$ & $\begin{array}{l}\text { Principaux } \\
\text { résultats }\end{array}$ \\
\hline $\begin{array}{l}\text { Montebello, Saias, } \\
\text { Greffeuille (1974) }\end{array}$ & 24 & Industrie & I & $\begin{array}{l}\text { Quantitative } \\
\text { Statistique }\end{array}$ & $\begin{array}{l}\text { Indétermination du lien PS- } \\
\text { performance. }\end{array}$ \\
\hline Robinson (1979) & 42 & Services & $\mathrm{CA}<150000$ & $\begin{array}{l}\text { Qualitative } \\
\text { Interventions }\end{array}$ & $\begin{array}{l}\text { PS améliore le processus de } \\
\text { prise de décision. }\end{array}$ \\
\hline Unni (1981) & 120 & Divers & I & $\begin{array}{l}\text { Quantitative } \\
\text { Statistique }\end{array}$ & $\begin{array}{l}\text { PS en soi n'est pas un gage } \\
\text { de succès pour la PME. }\end{array}$ \\
\hline Robinson (1982) & 101 & $\begin{array}{l}\text { Services } \\
\text { Détail } \\
\text { Industrie }\end{array}$ & $\begin{array}{c}<50 \text { sal. } \\
\mathrm{CA}<3 \mathrm{M} \$\end{array}$ & $\begin{array}{l}\text { Quantitative } \\
\text { Statistique }\end{array}$ & $\begin{array}{l}\text { PME engagées dans PS } \\
\text { reposant sur aide } \\
\text { «outsiders » sont plus } \\
\text { efficaces que les autres. }\end{array}$ \\
\hline $\begin{array}{l}\text { Robinson et Pearce } \\
(1983)\end{array}$ & 51 & Banques & I & $\begin{array}{l}\text { Quantitative } \\
\text { Statistique }\end{array}$ & $\begin{array}{l}\text { La PS n'accroît pas la } \\
\text { performance, processus } \\
\text { formel ou pas. }\end{array}$ \\
\hline $\begin{array}{l}\text { Robinson et al. } \\
\text { (1984) }\end{array}$ & 51 & $\begin{array}{l}\text { Détail } \\
\text { Services }\end{array}$ & $\begin{array}{c}<50 \text { sal. } \\
\mathrm{CA}<3 \mathrm{M} \$\end{array}$ & $\begin{array}{l}\text { Quantitative } \\
\text { Statistique }\end{array}$ & $\begin{array}{l}\text { Impact de la PS positif sur la } \\
\text { performance des PME, quel } \\
\text { que soit le stade de } \\
\text { développement. }\end{array}$ \\
\hline Orpen (1985) & 52 & Divers & & $\begin{array}{l}\text { Quantitative } \\
\text { Questionnaires }\end{array}$ & $\begin{array}{l}\text { Performance accrue par PS si } \\
\text { la planification est de qualité. }\end{array}$ \\
\hline $\begin{array}{l}\text { Sexton et Van } \\
\text { Auken (1985) }\end{array}$ & 357 & Divers & I & $\begin{array}{l}\text { Qualitative } \\
\text { Longitudinale } \\
\text { Entretiens }\end{array}$ & $\begin{array}{l}\text { PS importante pour la survie } \\
\text { des PME. }\end{array}$ \\
\hline $\begin{array}{l}\text { Ackelsberg et } \\
\text { Arlow (1985) }\end{array}$ & 135 & I & I & $\begin{array}{l}\text { Quantitative } \\
\text { Statistique }\end{array}$ & $\begin{array}{l}\text { Les PME peuvent bénéficier } \\
\text { d'une planification. }\end{array}$ \\
\hline $\begin{array}{l}\text { Bracker et Pearson } \\
(1986)\end{array}$ & 188 & Services & $\begin{array}{c}\mathrm{CA}<5 \mathrm{M} \$ \\
>5 \text { ans } \\
\text { d'existence }\end{array}$ & $\begin{array}{l}\text { Quantitative } \\
\text { Statistique }\end{array}$ & $\begin{array}{l}\text { Plans structurés plus } \\
\text { performants qu'autres types } \\
\text { de planification. }\end{array}$ \\
\hline $\begin{array}{l}\text { Robinson, Logan et } \\
\text { Salem (1986) }\end{array}$ & 81 & $\begin{array}{l}\text { Détail } \\
\text { aliments }\end{array}$ & I & $\begin{array}{l}\text { Quantitative } \\
\text { Statistique }\end{array}$ & $\begin{array}{l}\text { PO associée plus } \\
\text { significativement à la } \\
\text { performance que la PS. }\end{array}$ \\
\hline $\begin{array}{l}\text { Bracker, Keats et } \\
\text { Pearson (1988) }\end{array}$ & 73 & $\begin{array}{l}\text { Industrie } \\
\text { électro- } \\
\text { nique }\end{array}$ & $\begin{array}{l}5 \text { ans d'existence } \\
\quad<100 \text { sal. }\end{array}$ & $\begin{array}{l}\text { Quantitative } \\
\text { Statistique }\end{array}$ & $\begin{array}{l}\text { Lien PS-performance en } \\
\text { PME si procédures } \\
\text { structurées. }\end{array}$ \\
\hline $\begin{array}{l}\text { Baker, Addams et } \\
\text { Davis (1993) }\end{array}$ & 194 & & $\begin{array}{c}\text { «1989 INC. } 500 \\
\text { companies } »\end{array}$ & $\begin{array}{l}\text { Quantitative } \\
\text { Statistique }\end{array}$ & $\begin{array}{l}\text { PS formelle et plan écrit } \\
\text { peuvent jouer un rôle } \\
\text { important. }\end{array}$ \\
\hline
\end{tabular}


la planification est positivement et significativement associée à la performance en PME, sans avancer, toutefois, que la planification améliore la performance. Pour Kargar et Parnell (1996), la majorité des études empiriques sur les PME considèrent, en général, une seule dimension de mesure (présence ou absence de la planification, degré de formalisation, etc.) pour expliquer les variations de la performance organisationnelle. Ils estiment que l'utilisation d'indicateurs de finance et marketing comme mesure de la performance représente une partie seulement de la performance d'une organisation. Ces indicateurs reposent sur la façon dont l'activité s'est améliorée dans le passé, sous-entendant qu'un tel succès peut s'extrapoler dans le futur. Or, cette démarche leur paraît contradictoire avec les systèmes de planification à plusieurs dimensions décrits dans la littérature récente. Ils vont donc adopter une perspective plus large, considérant la satisfaction à l'égard de la planification :

- au regard des résultats financiers et concrets pouvant être rattachés au processus de planification;

- au regard de la contribution des efforts de planification stratégique pour accroître l'efficacité organisationnelle.

Finalement, après avoir mené une étude par questionnaires dans le secteur des banques et traité statistiquement les résultats, ces deux auteurs concluent qu'il y a une association positive entre les dimensions du processus de planification et les indicateurs de performance retenus. Cependant, l'hypothèse selon laquelle une grande importance accordée aux caractéristiques du processus de planification entraîne une grande satisfaction (et inversement) n'est que partiellement vérifiée.

Kargar (1996), passant en revue une variété d'études sur la planification en PME dans les années 1980-1990, souligne le fait que les recherches empiriques en PME utilisent, en général, une seule dimension (présence ou absence de la planification, degré de formalisation) pour expliquer les variations de performance, ce qui va à l'encontre, selon lui, de l'approche multidimensionnelle des systèmes de planification développés dans la littérature récente. De la même manière, les approches traditionnelles sur le lien planification-performance s'appuient sur des indicateurs financiers ou marketing de mesure de la performance, ce qui n'est qu'un élément de la performance organisationnelle.

Dès lors, sur la base d'une étude quantitative par questionnaires auprès de 41 banques, Kargar parvient aux propositions suivantes :

- selon une approche multivariée, il y a une forte relation entre les caractéristiques du système de planification et les deux premières perspectives de mesure de la performance (pas de prédominance de la performance financière);

Revue internationale P.M.E., vol. 14, n 2, 2001

(C) 2001 - Presses de l'Université du Québec

Édifice Le Delta I, 2875, boul. Laurier, bureau 450, Sainte-Foy, Québec G1V 2M2 • Tél. : (418) 657-4399 - www.puq.uquebec.ca

Tiré de : Revue internationale P.M.E., vol. $14, \mathrm{n}^{\circ} 2$, sous la direction de Pierre-André Julien. 
- le degré d'orientation externe du système et l'implication du personnel dans le processus de planification sont les deux facteurs les plus importants pour l'efficacité de la planification; viennent ensuite le niveau d'intégration et le degré d'orientation interne. L'utilisation de techniques analytiques semble être la dimension la moins importante.

De leur côté, Calori, Véry et Arrègle (1997) estiment que les divergences proviennent de la sous-estimation de paramètres de contingence, tels que la turbulence de l'environnement, la nature de l'activité, la complexité de l'entreprise ou la logique de l'entrepreneur. Ces trois auteurs se positionnent dans le courant de pensée dominant selon lequel la PME se caractérise par le rôle essentiel du dirigeant, tout à la fois entrepreneur, manager et organisateur: les objectifs poursuivis par l'entreprise correspondent à ceux du dirigeant. Leur principal résultat peut s'énoncer en s'inspirant du titre du dernier ouvrage de Mintzberg (1994) : il n'y a ni grandeur, ni décadence de la planification stratégique en PMI, tout dépend du contexte. En d'autres termes, la relation entre la planification en PMI et sa performance (ROI) est influencée par les variables de contingence énoncées ci-dessus.

Comme précédemment, le tableau 2 synthétise les principales études, permettant peut-être ainsi de mieux comprendre la contradiction de certains résultats au vu de la diversité des recherches.

TABleau 2

Études ayant tenté de conclure à propos de la valeur de la planification en PME

\begin{tabular}{|c|c|c|c|c|c|}
\hline $\begin{array}{l}\text { Auteurs } \\
\text { et année }\end{array}$ & $\begin{array}{l}\text { Firmes } \\
\text { étudiées }\end{array}$ & $\begin{array}{l}\text { Secteur } \\
\text { d'activité }\end{array}$ & $\begin{array}{l}\text { Définition de la } \\
\text { PME retenue }\end{array}$ & $\begin{array}{l}\text { Méthodologie } \\
\text { employée }\end{array}$ & $\begin{array}{l}\text { Principaux } \\
\text { résultats }\end{array}$ \\
\hline $\begin{array}{l}\text { Shrader, Mulford e } \\
\text { Blackburn (1989) }\end{array}$ & et & $\begin{array}{l}\text { Industrie } \\
\text { Vente au } \\
\text { détail } \\
\text { Services }\end{array}$ & $<100$ salariés & $\begin{array}{l}\text { Quantitative } \\
\text { Statistique }\end{array}$ & $\begin{array}{l}\text { Lien positif entre PS et } \\
\text { performance en général. }\end{array}$ \\
\hline $\begin{array}{l}\text { Schwenk et } \\
\text { Shrader (1993) an }\end{array}$ & $\begin{array}{l}\text { « Méta- } \\
\text { analyse » }\end{array}$ & Divers & $<100$ salariés & $\begin{array}{l}\text { Quantitative } \\
\text { Statistique }\end{array}$ & $\begin{array}{l}\text { Association positive et } \\
\text { significative entre PS et } \\
\text { performance en PME. }\end{array}$ \\
\hline Kargar (1996) & 41 & Banques & $\begin{array}{c}>5 \text { ans } \\
\text { d'existence }\end{array}$ & $\begin{array}{l}\text { Quantitative } \\
\text { Statistique }\end{array}$ & $\begin{array}{l}\text { Intérêt de la PS plus au } \\
\text { niveau processus que } \\
\text { rentabilité. }\end{array}$ \\
\hline $\begin{array}{l}\text { Kargar et Parnell } \\
\text { (1996) }\end{array}$ & 41 & $\begin{array}{l}\text { Banques } \\
\text { com- } \\
\text { merciales }\end{array}$ & I & $\begin{array}{l}\text { Quantitative } \\
\text { Statistique }\end{array}$ & $\begin{array}{l}\text { PS efficace positivement } \\
\text { corrélée à la performance. }\end{array}$ \\
\hline $\begin{array}{l}\text { Calori, Véry et } \\
\text { Arrègle (1997) }\end{array}$ & 1589 & $\begin{array}{l}\text { Biens } \\
\text { profes- } \\
\text { sionnels } \\
\end{array}$ & $<500$ employés & $\begin{array}{l}\text { Quantitative } \\
\text { Statistique }\end{array}$ & $\begin{array}{l}\text { Ni grandeur ni décadence de } \\
\text { la PS en PME: tout dépend } \\
\text { du contexte. }\end{array}$ \\
\hline $\begin{array}{l}\text { Berman, Gordon et } \\
\text { Sussman (1997) }\end{array}$ & 161 & 1 & I & $\begin{array}{l}\text { Quantitative } \\
\text { Statistique }\end{array}$ & $\begin{array}{l}\text { En général, les PME qui } \\
\text { planifient ont de meilleurs } \\
\text { résultats. }\end{array}$ \\
\hline
\end{tabular}


En conclusion de cette partie, il convient de souligner que les résultats contradictoires obtenus quant au lien entre planification et performance en PME ne sont pas spécifiques à ce type d'entreprises : la même divergence existe dans les travaux portant sur les grandes organisations. Par ailleurs, il nous semble que l'axe qui consiste à étudier la planification en PME par le biais de la relation planificationperformance est probablement le plus important, aussi bien en termes de nombre de recherches qu'en termes de résultats. Pour autant, il ne nous semble pas que cette approche soit la plus à même de fournir des conclusions intéressantes dans l'étude de la planification dans les petites et moyennes entreprises, tant nous avons l'impression qu'il serait difficile de contribuer par là même à un début de théorie de la planification en PME.

La question de la performance des organisations est délicate à aborder. Elle suppose, en effet, que sur un thème en particulier, comme celui de la planification par exemple, l'on fasse abstraction des autres facteurs susceptibles de l'influencer (toutes choses égales d'ailleurs). Or, en sciences de gestion, il semble toujours très réducteur d'avoir ce type d'approche. Par ailleurs, il nous apparaît qu'avant de s'interroger sur l'efficacité d'une pratique managériale il convient de bien saisir la réalité de cette pratique. Or, nous ne pensons pas que cela soit le cas concernant la connaissance actuelle sur le comportement planificateur des PME. Voyons, dès lors, dans quelle mesure on peut ne pas se satisfaire de ce constat.

\section{Les domaines de la recherche sur la planification des PME qui restent dans l'ombre}

Avant de s'interroger sur la valeur de la planification en PME, a-t-on vraiment répondu à la question de savoir quelles sont les démarches stratégiques en PME? La planification n'est pas une démarche isolée, mais s'inscrit dans un processus plus global de formation de la stratégie. Ne faudrait-il pas repartir de là ? Plus généralement, les questions fondamentales sont les suivantes :

- Quels sont les réels besoins des PME en matière de planification?

- Quels sont les déterminants de la planification en PME?

- Quels sont les acteurs de la stratégie en PME?

- Quels sont les processus de prise de décision en PME?

Dès lors, voyons dans quelle mesure l'état actuel de la recherche permet de répondre à ces interrogations capitales.

Revue internationale P.M.E., vol. 14, n 2, 2001

(C) 2001 - Presses de l'Université du Québec

Édifice Le Delta I, 2875, boul. Laurier, bureau 450, Sainte-Foy, Québec G1V 2M2 • Tél. : (418) 657-4399 - www.puq.uquebec.ca

Tiré de : Revue internationale P.M.E., vol. 14, n² , sous la direction de Pierre-André Julien. 


\subsection{Le courant de recherche descriptif sur la planification en PME, ou l'étude des pratiques}

Nombre d'études sur la planification en PME ont cherché à démontrer le caractère plus ou moins répandu de la planification dans les firmes de petite et moyenne dimension. Ces recherches ont globalement conclu à des pratiques différentes.

\subsubsection{Les apports de recherches empiriques}

La principale conclusion des recherches empiriques est que la planification n'est pas une pratique courante dans les firmes de petite et moyenne dimension (Bamberger, 1980 ; Robinson, 1982 ; Sexton et Van Auken, 1985 ; Shuman, Shaw et Sussman, 1985 ; Robinson, Logan et Salem, 1986; Shaw, Shuman et Sussman, 1986 ; Shrader, Mulford et Blackburn, 1989 ; Rousseau, 1992 ; Waalewijn et Segaar, 1993). Pour autant, lorsqu'elle existe, la planification en PME a des caractéristiques diverses. Le tableau 3 synthétise ces propositions.

TABleau 3

La planification en PME, une pratique peu courante

\begin{tabular}{|c|c|c|c|c|c|}
\hline $\begin{array}{l}\text { Auteurs } \\
\text { et année }\end{array}$ & $\begin{array}{l}\text { Firmes } \\
\text { étudiées }\end{array}$ & $\begin{array}{l}\text { Secteur } \\
\text { d'activité }\end{array}$ & $\begin{array}{l}\text { Définition de la } \\
\text { PME retenue }\end{array}$ & $\begin{array}{l}\text { Méthodologie } \\
\text { employée }\end{array}$ & $\begin{array}{l}\text { Principaux } \\
\text { résultats }\end{array}$ \\
\hline Bamberger (1980) & I & I & $<500$ salariés & $\begin{array}{l}\text { Qualitative } \\
\text { Entretiens }\end{array}$ & $\begin{array}{l}\text { Méthodes de décision et de } \\
\text { planification peu utilisées } \\
(24 \%) \text {. }\end{array}$ \\
\hline $\begin{array}{l}\text { Sexton et Van } \\
\text { Auken (1982) }\end{array}$ & 357 & Divers & I & $\begin{array}{l}\text { Quantitative } \\
\text { Statistique }\end{array}$ & $\begin{array}{l}\text { Réflexion stratégique dans } \\
25 \% \text { des cas, sans plans } \\
\text { d'action. }\end{array}$ \\
\hline $\begin{array}{l}\text { Sexton et Van } \\
\text { Auken (1985) }\end{array}$ & 357 & Divers & I & $\begin{array}{l}\text { Qualitative } \\
\text { Entretiens sur } \\
3 \text { ans } \\
\end{array}$ & $\begin{array}{l}\text { PS utilisée par minorité de } \\
\text { PME et sporadiquement. }\end{array}$ \\
\hline $\begin{array}{l}\text { Shuman, Shaw et } \\
\text { Sussman (1985) }\end{array}$ & 220 & Divers & $\begin{array}{l}\text { «1983 INC. } 500 \\
\text { companies » }\end{array}$ & $\begin{array}{l}\text { Quantitative } \\
\text { Statistique }\end{array}$ & $\begin{array}{l}\text { Plupart des PME adopte une } \\
\text { forme de PS après } \\
\text { démarrage. }\end{array}$ \\
\hline $\begin{array}{l}\text { Shaw, Shuman et } \\
\text { Sussman (1986) }\end{array}$ & 135 & Divers & l & $\begin{array}{l}\text { Quantitative } \\
\text { Statistique }\end{array}$ & $\begin{array}{l}30 \% \text { des PME ont recours à } \\
\text { la PS. }\end{array}$ \\
\hline $\begin{array}{l}\text { Robinson, Logan et } \\
\text { Salem (1986) }\end{array}$ & 81 & $\begin{array}{l}\text { Détail } \\
\text { alimentaire }\end{array}$ & I & $\begin{array}{l}\text { Quantitative } \\
\text { Statistique }\end{array}$ & $\begin{array}{l}\text { Planification opérationnelle } \\
\text { plus importante que PS. }\end{array}$ \\
\hline $\begin{array}{l}\text { Shrader, Mulford, } \\
\text { Blackburn (1989) }\end{array}$ & 97 & $\begin{array}{l}\text { Industrie } \\
\text { Vente } \\
\text { Services }\end{array}$ & $<100$ salariés & $\begin{array}{l}\text { Quantitative } \\
\text { Statistique }\end{array}$ & $\begin{array}{l}\text { PO plus répandue et utilisée } \\
\text { que PS. }\end{array}$ \\
\hline Rousseau (1992) & 35 & Industrie & 1 & $\begin{array}{l}\text { Quantitative } \\
\text { Statistique }\end{array}$ & $\begin{array}{l}\text { Un tiers des PME font de la } \\
\text { PS. }\end{array}$ \\
\hline $\begin{array}{l}\text { Waalewijn et } \\
\text { Segaar (1993) }\end{array}$ & 200 & I & $\begin{array}{l}\text { Firmes moyennes } \\
\quad>100 \text { sal. }\end{array}$ & $\begin{array}{l}\text { Quantitative } \\
\text { Statistique }\end{array}$ & $\begin{array}{l}\text { Faiblesse de la PS en PME } \\
(20 \%) \text { mais pratique des } \\
\text { budgets. }\end{array}$ \\
\hline
\end{tabular}


De même, pour la plupart des chercheurs, les activités de planification sont de nature informelle et non structurée dans les PME (Shuman, 1975; Fankam, 1998), ce qui s'estompe pour Shuman, Shaw et Sussman (1985), qui pensent à ce propos que le processus de planification utilisé devient plus formel et structuré avec la croissance des entreprises. Sexton et Dahle (1976) ne retrouvent pas, dans le cadre de la PME, le processus de la planification à long terme académique, bien que certains de ses éléments soient pris en considération dans la charpente de la prise de décision de ces firmes, moins sophistiquée que dans les grandes entreprises. Bhatty (1981) estime, pour sa part, la nécessité de considérer une approche contingente de la planification en moyenne entreprise, en fonction du degré de changement et de la complexité de l'environnement.

Le caractère non systématique de la planification en PME est un aspect souvent relevé dans les études (Robinson, 1982 ; Robinson, Logan et Salem, 1986), tout comme sa nature sporadique (Sexton et Van Auken, 1985). Globalement, Shrader, Mulford et Blackburn (1989) estiment que la planification dans les petites et moyennes entreprises s'apparente à du «tout ou rien» (pas de plans écrits ou planification sophistiquée). Ces propositions sont synthétisées dans le tableau 4.

TABLEAU 4

\section{Le caractère peu analytique de la planification stratégique en PME}

\begin{tabular}{|c|c|c|c|c|c|}
\hline $\begin{array}{l}\text { Auteurs } \\
\text { et année }\end{array}$ & $\begin{array}{l}\text { Firmes } \\
\text { étudiées }\end{array}$ & $\begin{array}{l}\text { Secteur } \\
\text { d'activité }\end{array}$ & $\begin{array}{l}\text { Définition de la } \\
\text { PME retenue }\end{array}$ & $\begin{array}{l}\text { Méthodologie } \\
\text { employée }\end{array}$ & $\begin{array}{l}\text { Principaux } \\
\text { résultats }\end{array}$ \\
\hline Shuman (1975) & 41 & Industrie & $\begin{array}{l}\text { Jusqu'à } 800 \\
\text { salariés }\end{array}$ & $\begin{array}{l}\text { Quantitative } \\
\text { Statistique }\end{array}$ & $\begin{array}{l}\text { PS informelle, non structurée } \\
\text { et sporadique. }\end{array}$ \\
\hline $\begin{array}{l}\text { Sexton et Dahle } \\
\text { (1976) }\end{array}$ & 20 & Commerce & I & $\begin{array}{l}\text { Qualitative } \\
\text { Interviews }\end{array}$ & $\begin{array}{l}\text { PLT plus répandue qu'a } \\
\text { priori, malgré une faible } \\
\text { sophistication. }\end{array}$ \\
\hline Bhatty (1981) & 26 & Divers & $\begin{array}{l}\text { Entre } 1800 \\
\text { et } 4999 \\
\text { salariés }\end{array}$ & $\begin{array}{l}\text { Quantitative } \\
\text { Statistique }\end{array}$ & $\begin{array}{l}\text { PS formelle répandue dans } \\
\text { les entreprises moyennes, sur } \\
\text { trois ans. }\end{array}$ \\
\hline $\begin{array}{l}\text { Baker, Addams et } \\
\text { Davis (1993) }\end{array}$ & 194 & 1 & $\begin{array}{l}\text { «1989 INC. } 500 \\
\text { companies } »\end{array}$ & $\begin{array}{l}\text { Quantitative } \\
\text { Statistique }\end{array}$ & $\begin{array}{l}\text { Firmes à croissance rapide } \\
\text { mettent en œuvre PS, plans } \\
\text { écrits. }\end{array}$ \\
\hline $\begin{array}{l}\text { Calori, Véry et } \\
\text { Arrègle (1997) }\end{array}$ & 1589 & $\begin{array}{l}\text { Biens } \\
\text { profes- } \\
\text { sionnels, } \\
\text { intermé- } \\
\text { diaires }\end{array}$ & $<500$ employés & $\begin{array}{l}\text { Quantitative } \\
\text { Statistique }\end{array}$ & $\begin{array}{l}30 \% \text { des PME pratiquent la } \\
\text { PS (anticipation et } \\
\text { formalisation fortes). }\end{array}$ \\
\hline Fankam (1998) & 220 & Industrie & $<500$ salariés & $\begin{array}{l}\text { Quantitative } \\
\text { Statistique }\end{array}$ & $\begin{array}{l}\text { Peu de formalisation en } \\
\text { PME. }\end{array}$ \\
\hline
\end{tabular}




\subsubsection{La planification en PME : un acte manqué?}

Pour Rice (1983), le manque de planification en PME (son résultat principal, avons-nous constaté) est généralement attribué à l'ignorance des diverses approches stratégiques, d'une part, ainsi qu'à l'incapacité des dirigeants de PME à exercer une analyse de la décision sophistiquée, d'autre part (Thomas, 1971; Robinson, 1982). Pour autant, les théoriciens de la PME encouragent les dirigeants à adopter les pratiques des grandes entreprises (méthodes d'évaluation formalisées, analyses statistiques, intégration des facteurs dans un plan détaillé, etc.). Mais Rice se pose la question suivante : le fait que les dirigeants de PME ne font pas ce type de planification élaborée peut-il nous autoriser à les accuser de gérer leurs affaires sans se préoccuper de l'évolution à long terme ? Selon lui, la question pourrait être inversée : les PME ne font pas de planification élaborée, car elles n'en éprouvent pas le besoin, à l'inverse des grandes entreprises. Dès lors, de l'importance accordée aux dimensions de la prise de décisions stratégiques en PME, il est possible de déduire que la planification a réellement lieu, même si elle n'est pas approfondie, formalisée et précise (Robinson et Pearce, 1983).

Ainsi, il n'y a pas une approche unique et impérative de la planification en PME (ce qui est également le cas dans les grandes organisations d'ailleurs), ce qui peut contribuer à expliquer les variations dans les résultats d'études empiriques, ayant pour source des prises de position trop rigides et catégoriques à l'égard de l'appréhension de la planification dans les firmes de petite et moyenne dimension. Le tableau 5 fait ressortir l'hétérogénéité des approches retenues, aussi bien en termes de méthodologie employée que de secteur d'activité considéré, ou encore de définition donnée à la PME.

Il nous semble que la grande majorité des études évoquées ci-dessus ne peuvent que difficilement saisir la réalité des pratiques de planification dans les firmes de petite et moyenne dimension du fait, notamment, d'une définition de la planification a priori reposant sur les développements de cette notion relatifs à la grande entreprise (ce qui est notamment le cas de l'étude récente de Calori, Véry et Arrègle, 1997), mais aussi du choix d'une méthodologie quantitative, selon une logique hypothéticodéductive, pas toujours appropriée, selon nous, pour caractériser le comportement planificateur des PME. Voyons dès à présent la nature des résultats des recherches relatives à l'étude des processus de planification dans les PME.

\subsection{Le courant de recherche normatif sur la planification en PME, ou l'étude des processus}

De façon générale, la planification en PME, appréhendée comme un processus de gestion, a des racines conceptuelles dans la littérature relative à la grande entreprise. Mais ces développements, s'ils adhèrent aux modèles de planification prescrits dans la littérature en management stratégique, sont réalisés dans un contexte de firme 
TABLEAU 5

Recherches soulignant l'hétérogénéité des approches

\begin{tabular}{|c|c|c|c|c|c|}
\hline $\begin{array}{l}\text { Auteurs } \\
\text { et année }\end{array}$ & $\begin{array}{l}\text { Firmes } \\
\text { étudiées }\end{array}$ & $\begin{array}{l}\text { Secteur } \\
\text { d'activité }\end{array}$ & $\begin{array}{l}\text { Définition de la } \\
\text { PME retenue }\end{array}$ & $\begin{array}{l}\text { Méthodologie } \\
\text { employée }\end{array}$ & $\begin{array}{l}\text { Principaux } \\
\text { résultats }\end{array}$ \\
\hline $\begin{array}{l}\text { Montebello, Saias } \\
\text { et Greffeuille } \\
\text { (1974) }\end{array}$ & 24 & Industrie & I & $\begin{array}{l}\text { Quantitative } \\
\text { Statistique }\end{array}$ & $\begin{array}{l}\text { PE comme PME peuvent } \\
\text { adopter un comportement } \\
\text { planificateur. }\end{array}$ \\
\hline $\begin{array}{l}\text { Rice et Hamilton } \\
\text { (1979) }\end{array}$ & 35 & $\begin{array}{l}\text { Services, } \\
\text { grossistes } \\
\text { détaillants }\end{array}$ & $\begin{array}{l}\text { Entre } 1 \text { et } 190 \\
\text { employés }\end{array}$ & $\begin{array}{l}\text { Qualitative } \\
\text { Entretiens }\end{array}$ & $\begin{array}{l}\text { PS non rationnelle et non } \\
\text { systématique en PME. }\end{array}$ \\
\hline Robinson (1982) & 101 & $\begin{array}{l}\text { Services } \\
\text { Détail } \\
\text { Industrie }\end{array}$ & $\begin{array}{c}<50 \mathrm{sal} \\
\mathrm{CA}<3 \mathrm{M} \$\end{array}$ & $\begin{array}{l}\text { Quantitative } \\
\text { Statistique }\end{array}$ & $\begin{array}{l}\text { Nécessité d'inclure des } \\
\text { «outsiders » dans l'effort de } \\
\text { PS des PME. }\end{array}$ \\
\hline Jones (1982) & 69 & Services & I & $\begin{array}{l}\text { Quantitative } \\
\text { Enquête }\end{array}$ & $\begin{array}{l}14 \text { variables qui différencient } \\
\text { les planificateurs des non- } \\
\text { planificateurs. }\end{array}$ \\
\hline Rice (1983) & 22 & Divers & I & $\begin{array}{l}\text { Qualitative } \\
\text { Entretiens }\end{array}$ & $\begin{array}{l}\text { Existence d'une PS } \\
\text { informelle, fragmentée, } \\
\text { incrémentale et heuristique } \\
\text { en PME. }\end{array}$ \\
\hline $\begin{array}{l}\text { Robinson et al. } \\
\text { (1986) }\end{array}$ & 81 & Détail & Indépendance & $\begin{array}{l}\text { Quantitative } \\
\text { Statistique }\end{array}$ & $\begin{array}{l}\text { Liste de variables dans la PS } \\
\text { (estimation des ventes, } \\
\text { analyse de la concurrence, } \\
\text { etc.). }\end{array}$ \\
\hline
\end{tabular}

de taille importante et restent adaptés à ce champ. Ainsi, les chercheurs qui se sont intéressés à la question des processus de planification dans les firmes de petite et moyenne dimension ont-ils été amenés à proposer des modèles «simplifiés », sans consensus de leur part.

\subsubsection{Les caractéristiques d'un processus de planification en PME}

Nombre de chercheurs ont contribué à l'analyse des processus de planification dans les firmes de petite et moyenne dimension. Parmi eux, Scarborough et Zimmerer (1987) estiment qu'en raison de leur taille et de caractéristiques particulières (pauvreté en ressources, style de gestion flexible, structure organisationnelle informelle, adaptation au changement) les PME ont besoin d'une approche spécifique de la planification stratégique :

- un horizon de la planification assez court (deux ans maximum);

- un processus informel et peu structuré (approche «shirt-sleeve»);

- une procédure qui doit tenir compte d'acteurs externes pour améliorer la fiabilité et la créativité du plan résultant;

- un processus qui ne doit pas commencer par l'établissement d'objectifs.

Revue internationale P.M.E., vol. 14, $\mathrm{n}^{\circ}$ 2, 2001 
Pour leur part, Berman, Gordon et Sussman (1997) avancent que l'on peut espérer trouver une large variété de dimensions de processus de planification en PME :

- le plus commun d'entre eux réside dans l'utilisation de buts et d'objectifs (Robinson et Pearce, 1983 ; Shrader, Mulford et Blackburn, 1989);

- un autre aspect du processus de planification étudié est la prévision (Robinson et Pearce, 1983);

- une variable utilisée pour mesurer le processus de planification en PME est l'existence de plans écrits (Ackelsberg et Arlow, 1985; Robinson et Pearce, 1983 ; Shrader, Mulford et Blackburn, 1989).

Le tableau 6 regroupe les principaux travaux sur ce thème.

TABLEAU 6

Travaux traitant du processus de planification stratégique en PME

\begin{tabular}{|c|c|c|c|c|c|}
\hline $\begin{array}{l}\text { Auteurs } \\
\text { et année }\end{array}$ & $\begin{array}{l}\text { Firmes } \\
\text { étudiées }\end{array}$ & $\begin{array}{l}\text { Secteur } \\
\text { d'activité }\end{array}$ & $\begin{array}{l}\text { Définition de la } \\
\text { PME retenue }\end{array}$ & $\begin{array}{l}\text { Méthodologie } \\
\text { employée }\end{array}$ & $\begin{array}{l}\text { Principaux } \\
\text { résultats }\end{array}$ \\
\hline Marmuse (1979) & 30 & 1 & $\begin{array}{l}<500 \text { salariés } \\
\mathrm{CA}<100 \mathrm{MF}\end{array}$ & $\begin{array}{l}\text { Étude } \\
\text { prescriptive } \\
\text { Consultations }\end{array}$ & $\begin{array}{l}\text { Mise en œuvre PS doit tenir } \\
\text { compte des contraintes } \\
\text { marché/structure } \\
\text { financière/coûts et dualité } \\
\text { des objectifs. }\end{array}$ \\
\hline Van Hoorn (1979) & 75 & $\begin{array}{l}\text { Industrie } \\
\end{array}$ & $\begin{array}{c}50<\text { effectif }<500 \\
3 \mathrm{M} \$<\mathrm{CA}<100 \mathrm{M} \\
\$\end{array}$ & $\begin{array}{l}\text { Qualitative } \\
\text { Entretiens }\end{array}$ & $\begin{array}{l}\text { Processus de PS relève à la } \\
\text { fois de la créativité et du } \\
\text { contrôle. }\end{array}$ \\
\hline $\begin{array}{l}\text { Robinson et Pearce } \\
\text { (1983) }\end{array}$ & 51 & Banques & I & $\begin{array}{l}\text { Quantitative } \\
\text { Statistique }\end{array}$ & $\begin{array}{l}\text { Égale importance accordée } \\
\text { aux dimensions de la prise de } \\
\text { décision stratégique, formelle } \\
\text { ou non. }\end{array}$ \\
\hline Orpen (1985) & 52 & Divers & I & $\begin{array}{l}\text { Quantitative } \\
\text { Question- } \\
\text { naires }\end{array}$ & $\begin{array}{l}\text { Qualité de la PS primordiale } \\
\text { (formalisation, rninutie, etc.). }\end{array}$ \\
\hline $\begin{array}{l}\text { Bracker, Keats et } \\
\text { Pearson (1988) }\end{array}$ & 73 & $\begin{array}{l}\text { Industrie } \\
\text { électronique }\end{array}$ & $\begin{array}{l}5 \text { ans d'existence } \\
\quad<100 \text { sal. }\end{array}$ & $\begin{array}{l}\text { Quantitative } \\
\text { Statistique }\end{array}$ & $\begin{array}{l}\text { Importance du degré de } \\
\text { sophistication du processus } \\
\text { de PS en PME. }\end{array}$ \\
\hline Kargar (1996) & 41 & Banques & $\begin{array}{l}\text { Plus de } 5 \text { ans } \\
\text { d'existence }\end{array}$ & $\begin{array}{l}\text { Quantitative } \\
\text { Statistique }\end{array}$ & $\begin{array}{l}\text { Degré d'orientation externe } \\
\text { et implication du personnel } \\
\text { comme facteurs importants } \\
\text { dans processus. }\end{array}$ \\
\hline
\end{tabular}

Les différents facteurs relatifs aux processus de planification évoqués ainsi que l'effet modérateur de certaines variables sur le processus souligné participent globalement aux différents modèles proprement dits de planification proposés dans la littérature, auxquels il convient de s'attarder à présent. 


\subsubsection{Quelques modèles de planification pour les PME issus de la littérature}

Pour Bamberger (1979), «le processus de planification politique en PME peut reposer sur la même logique fondamentale que le modèle normatif des sciences de la planification, mais le processus et son output seront moins détaillés et formalisés ». Ainsi, plusieurs chercheurs ont proposé des méthodes de planification qu'ils jugent eux-mêmes simples et pratiques. C'est le cas de Gilmore (1971), pour qui le processus de formulation stratégique en PME peut être mené en six étapes successives (consigner la stratégie effective, identifier les problèmes, découvrir le noyau des problèmes, formuler des alternatives, évaluer les alternatives et, enfin, choisir la nouvelle stratégie). De la même manière, Forbes (1974) décline une proposition en 10 étapes (analyse des frontières de l'activité actuelle, analyse des résultats liés à l'activité, liste des facteurs clés qui influencent l'activité, approximation des évolutions probables des facteurs clés, identification des valeurs des propriétaires et principaux dirigeants, établissement d'objectifs, estimation des forces et faiblesses, mise en place d'un programme d'action, détermination des priorités et estimation de sa propre position). Sans préciser les différentes étapes préconisées, d'autres auteurs ont également proposé leur approche du processus de planification stratégique en PME (Wheelwright, 1971 ; Bracker et Pearson, 1986; Scarborough et Zimmerer, 1987 ; Baker, Addams et Davis, 1993).

L'ensemble de ces contributions, au caractère normatif affirmé, constitue un apport d'une richesse intéressante, malgré leur variété rendant l'effort de synthèse difficile. Cependant, force est de constater que nous ne sommes pas très éloigné de la démarche LCAG des années 1960. Partant de ce constat d'absence de consensus dans la littérature sur l'élaboration du plan stratégique, ainsi que de manque d'une méthode d'adaptation d'un modèle quelconque aux exigences d'une PME, d'Amboise (1989) propose une synthèse remarquable des modèles de planification émergents de la littérature. Malgré ses qualités de synthèse, son apport ne se démarque pourtant pas des autres, selon nous, sur le plan de la contribution à une théorisation de la planification en PME. En effet, l'ensemble des études évoquées reste très prescriptif quant à la (bonne) méthode à employer afin de développer la planification stratégique dans les PME et, sans doute, pas assez descriptive des comportements planificateurs de ces firmes. Il nous semble que le problème réside une fois encore dans l'approche a priori retenue par la grande majorité des chercheurs qui s'intéressent à la planification des PME, fondée sur la littérature traitant de ce thème en grande entreprise. Suffirait-il dès lors de préconiser des modèles simplifiés de planification aux PME (voire simplistes), au lieu de

chercher à étudier la réalité des processus de planification tels qu'ils existent dans ces firmes?

Revue internationale P.M.E., vol. 14, $\mathrm{n}^{\circ}$ 2, 2001

(C) 2001 - Presses de l'Université du Québec

Édifice Le Delta I, 2875, boul. Laurier, bureau 450, Sainte-Foy, Québec G1V 2M2 - Tél. : (418) 657-4399 - www.puq.uquebec.ca

Tiré de : Revue internationale P.M.E., vol. $14, \mathrm{n}^{\circ} 2$, sous la direction de Pierre-André Julien. 
Comme précédemment, le tableau 7 illustre l'hétérogénéité des études empiriques sur le thème des processus de planification en PME, afin que le lecteur puisse fonder sa propre opinion sur l'état actuel de la recherche sur cette question.

TABLEAU 7

Diversité des études prescrivant un modèle de planification en PME

\begin{tabular}{|c|c|c|c|c|c|}
\hline $\begin{array}{l}\text { Auteurs } \\
\text { et année }\end{array}$ & $\begin{array}{l}\text { Firmes } \\
\text { étudiées }\end{array}$ & $\begin{array}{l}\text { Secteur } \\
\text { d'activité }\end{array}$ & $\begin{array}{l}\text { Définition de la } \\
\text { PME retenue }\end{array}$ & $\begin{array}{l}\text { Méthodologie } \\
\text { employée }\end{array}$ & $\begin{array}{l}\text { Principaux } \\
\text { résultats }\end{array}$ \\
\hline Wheelwright (1971) & 3 & Industrie & & $\begin{array}{l}\text { Étude } \\
\text { prescriptive } \\
\text { Consultations }\end{array}$ & $\begin{array}{l}\text { Proposition d'une procédure } \\
\text { de PS (identifier les variables } \\
\text { clés jusqu'à la mise par écrit } \\
\text { du plan). }\end{array}$ \\
\hline $\begin{array}{l}\text { Shuman, Shaw et } \\
\text { Sussman (1985) }\end{array}$ & 220 & Divers & $\begin{array}{c}\text { «1983 INC. } 500 \\
\text { companies } ~\end{array}$ & $\begin{array}{l}\text { Quantitative } \\
\text { Statistique }\end{array}$ & $\begin{array}{l}\text { Description approche } \\
\text { «standard» de PS en PME. }\end{array}$ \\
\hline $\begin{array}{l}\text { Bracker et Pearson } \\
\text { (1986) }\end{array}$ & 188 & Services & $\begin{array}{c}\mathrm{CA}<5 \mathrm{M} \$ \\
\text { Plus de } 5 \text { ans } \\
\text { d'existence }\end{array}$ & $\begin{array}{l}\text { Quantitative } \\
\text { Statistique }\end{array}$ & $\begin{array}{l}\text { Processus de PS est un } \\
\text { continuum de sophistication } \\
\text { en PME. }\end{array}$ \\
\hline $\begin{array}{l}\text { Baker, Addams et } \\
\text { Davis (1993) }\end{array}$ & 194 & & $\begin{array}{l}\text { «1989 INC. } 500 \\
\text { companies } »\end{array}$ & $\begin{array}{l}\text { Quantitative } \\
\text { Statistique }\end{array}$ & $\begin{array}{l}\text { Préconisation d'une approche } \\
\text { en quatre phases (plan } \\
\text { stratégique jusqu'à révision } \\
\text { formelle). }\end{array}$ \\
\hline $\begin{array}{l}\text { Kargar et Parnell } \\
(1996)\end{array}$ & 41 & $\begin{array}{l}\text { Banques } \\
\text { com- } \\
\text { merciales }\end{array}$ & & $\begin{array}{l}\text { Quantitative } \\
\text { Statistique }\end{array}$ & $\begin{array}{l}\text { PS doit être partie intégrante } \\
\text { du processus de MS des } \\
\text { PME. }\end{array}$ \\
\hline $\begin{array}{l}\text { Berman, Gordon et } \\
\text { Sussman (1997) }\end{array}$ & 161 & & & $\begin{array}{l}\text { Quantitative } \\
\text { Statistique }\end{array}$ & $\begin{array}{l}\text { Manque de processus } \\
\text { organisé et structuré en PME. }\end{array}$ \\
\hline
\end{tabular}

Les éléments proposés restent très intéressants dans l'absolu, sur un thème difficile à traiter selon nous. Pour autant, il nous semble qu'une fois encore les recherches traitant de l'analyse des processus de planification en PME, considérées globalement, n' aboutissent pas à un corpus de connaissances facilement exploitable. L'agglomération de chacun de ces travaux ne permet pas, en effet, de tendre vers une conceptualisation de la planification dans les PME.

\section{Conclusion}

Cet article ne serait sans doute pas complet sans laisser entrevoir les perspectives de recherche qui s'ouvrent à présent sur l'étude de la planification des PME. Comme nous avons pu le constater à travers ces développements, la question de l'étude de la planification en PME ne semble pas aboutie, loin s'en faut. Globalement, sur le thème de la planification dans les entreprises de petite et moyenne dimension, nous adhérons à l'idée selon laquelle la recherche en management stratégique des PME n'a certainement pas fait le tour de la question de la planification. 
Ainsi, tout en considérant le corpus existant en matière de recherches sur la planification des PME, nous entendons proposer des voies de recherche différentes de celles empruntées jusqu'à présent. Nous recommandons, en effet, aux chercheurs de se détacher de la grande majorité des études relatives à la planification des PME, fondées sur une approche très quantitative de la question, comme en témoignent les tableaux de synthèse. Nous considérons, en effet, que la grande majorité des études réalisées jusqu'à présent ont retenu une définition a priori de la planification en PME, fondée généralement sur la littérature portant sur la grande firme. Dès lors, pourquoi s'étonner de la proposition selon laquelle la majorité des entreprises de petite et moyenne dimension ne pratique pas effectivement une forme de planification, définie selon des critères adaptés à la grande entreprise ? La planification en PME est généralement appréhendée par défaut, de la manière suivante:

- en grande entreprise, la planification semble être un processus formel, faisant l'objet d'un plan écrit et communiqué à l'ensemble de la firme. Comme ce n'est en général pas le cas en PME, on dit de ces dernières que leur processus de planification est informel;

- en grande entreprise, la planification est souvent réalisée de façon systématique, c'est-à-dire reconduite régulièrement. Ayant pu constater que ce n'était la plupart du temps pas le cas pour les PME, certains chercheurs ont qualifié leur planification de sporadique;

- en grande entreprise, la planification est en général fondée sur une approche holistique, englobant l'ensemble de la firme. Comme c'est peu le cas dans les PME qui planifient, on dit que leur planification est partielle.

L'approche qui consiste donc à se fonder sur la littérature traitant de la planification dans les grandes entreprises, pour ensuite analyser quantitativement les pratiques des PME nous paraît donc faussée dès le départ. Sans doute convient-il de se positionner dans le courant d'analyse de la dénaturation au sens de Torrès (1997), qui permet, selon lui, « de concilier à la fois un fort degré d'autonomie de recherche par rapport aux travaux sur la grande entreprise et un fort degré de généralisation (ou de réfutation) de ces résultats, sans toutefois faire de la spécificité de la PME une loi universelle ou un postulat».

Nous préconisons donc de mettre à contribution la richesse constituée par l'ensemble des travaux antérieurs sur la planification en PME afin d'améliorer l'état des connaissances sur ce thème, utilisant une approche plus qualitative pour compléter et enrichir ce qui a déjà été produit. Une telle perspective permettrait, notamment, d'amorcer des réponses aux interrogations énoncées dans cet article : quelles formes prennent les démarches de planification en PME ? Comment caractériser les processus de management planifié au sein de ces firmes ? Ainsi, sans parler de recherche exploratoire en la matière, mais dans une perspective principalement 
inductive et dans un objectif de contribution à l'effort de théorisation de la planification en PME, il nous semble dès lors opportun de suivre un cheminement résolument différent (sans toutefois ignorer la littérature existante), illustré par la partie hachurée sur la figure 1.

FIGURE 1

\section{Cheminement de recherche proposé}

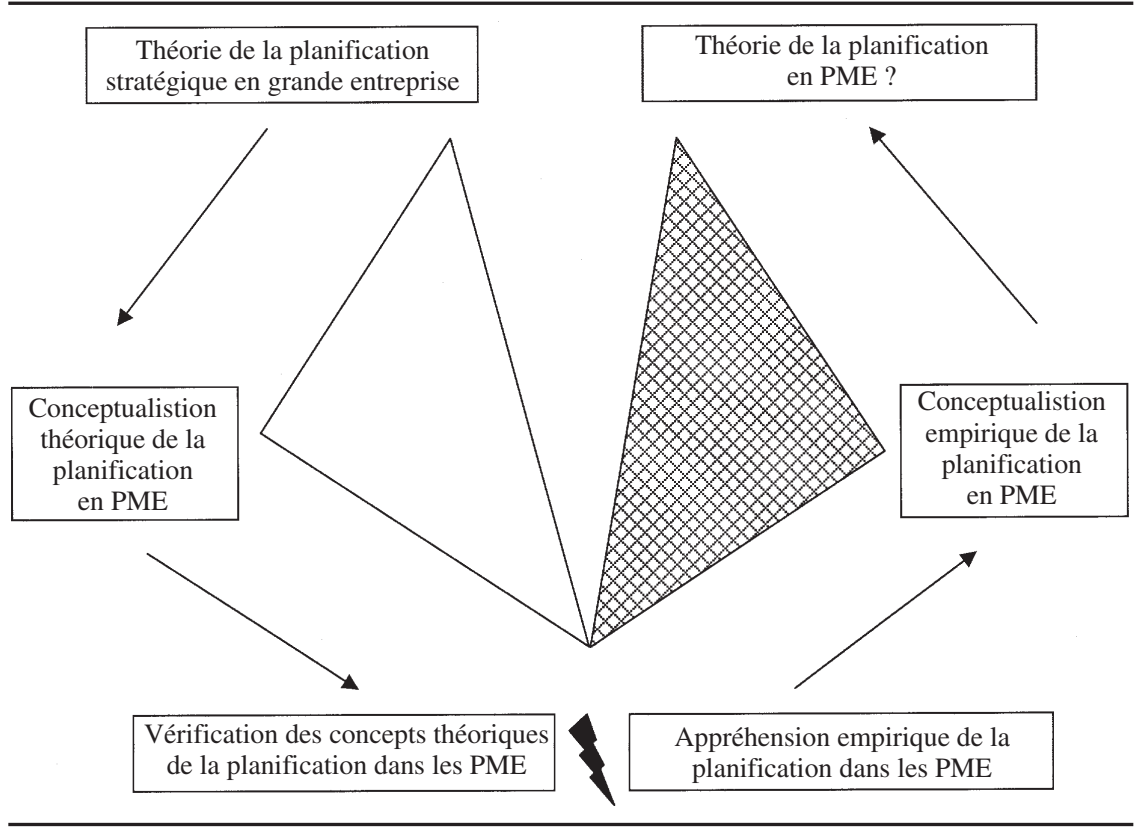

L'ancrage du chercheur dans les réalités des PME nous paraît particulièrement décisif dans l'amélioration des connaissances sur la planification de ces entreprises. Une première piste consisterait à recourir à l'approche méthodologique des cas. Cette méthode, qui utilise l'étude en profondeur d'un seul objet complexe (ou d'un petit nombre), tel qu'une organisation ou un incident, permet en effet de faire des généralisations vers une théorie (Yin, 1984). De multiples entretiens sur le terrain pourraient ainsi constituer la matière première des cas, certains travaux récents allant en ce sens (voir, notamment, Goy, 2000).

Une méthode plus appropriée pour la construction théorique telle que nous l'envisageons (c'est-à-dire un concept avec des catégories, sous-catégories, variables et liens) réside sans doute dans la théorisation ancrée («grounded theorizing » au sens de Glaser et Strauss, 1967), qui repose sur l'étude et la comparaison de nombreux exemples de l'objet d'étude. Certains, comme d'Amboise et Nkongolo 
Bakenda (1997), militent tout particulièrement en faveur de l'utilisation de cette méthodologie à la production des connaissances sur les petites et moyennes entreprises. Les chercheurs qui utilisent cette méthode ont élaboré une procédure spécifique (voir notamment Strauss, 1987 ; Corbin et Strauss, 1990 ; Dougherty et Heller, 1994) qui permet d'arriver, à partir de l'analyse du contenu des entrevues, à élaborer des concepts ou des théories de processus complexes. En d'autres termes, il s'agit de procéder par de multiples itérations de comparaisons constantes, d'une part, entre les données empiriques elles-mêmes et, d'autre part, entre les données empiriques et la théorie existante. Dans une telle démarche, la théorie existante devient un instrument pour appuyer les découvertes faites sur les données empiriques plutôt qu'un objectif à atteindre.

Voilà donc quelques pistes de recherche à emprunter, si tant est que l'on veuille faire progresser la recherche en management stratégique sur la planification des firmes de petite et moyenne dimension.

\section{Bibliographie}

ACKELSBERG, R. et P. ARLOW (1985), « Small business do plan and it pays off », Long Range Planning, vol. 18, $\mathrm{n}^{\mathrm{0}}$ 5, p. 61-67.

BAKER, W.H., H.L. ADDAMS et B. DAVIS (1993), « Business planning in successful small firms », Long Range Planning, vol. 26, nº 6, p. 82-88.

BAMBERGER, I. (1979), «La planification de la politique de l'entreprise dans les PME», Direction et Gestion, n ${ }^{\circ}$ 5, p. 31-40.

BAMBERGER, I. (1980), « Situations et comportements stratégiques des petites et moyennes entreprises », Direction et Gestion, no 4, p. 21-30.

Berman, J.A., D.D. Gordon et G. Sussman (1997), « A study to determine the benefits small business firms derive from sophisticated planning versus less sophisticated types of planning », Journal of Business and Economic Studies, vol. 3, n $\mathrm{n}^{\circ}$ 3, p. 1-11.

BHATTY, E.F. (1981), « Corporate planning in medium-sized companies in the U.K. », Long Range Planning, vol. 14, $\mathrm{n}^{\mathrm{o}}$ 1, p. 60-72.

BRACKER, J.S. et J.N. PEARSON (1986), «Planning and financial performance of small, mature firms », Strategic Management Journal, vol. 7, n 6, p. 503-522.

BRACKER, J.S., B.Q. KEATS et J.N. PEARSON (1988), «Planning and financial performance among small firms in a growth industry », Strategic Management Journal, vol. 9, no 6, p. 591-603.

CALORI, R., P. VÉRY et J.L. ARRÈGLE (1997), «Les PMI face à la planification stratégique », Revue française de gestion, $\mathrm{n}^{\mathrm{o}} 112, \mathrm{p} .11-23$.

Corbin, J. et A. STRAuss (1990), «Grounded theory research: procedures, canons and evaluative criteria », Qualitative Sociology, vol. 13, n 1, p. 3-21.

D’Amboise, G. (1989), « Planification stratégique dans les PME : des modèles émergents de la littérature », Revue PMO, vol. 4, n 2, p. 46-57.

Revue internationale P.M.E., vol. 14, n 2, 2001

(C) 2001 - Presses de l'Université du Québec

Édifice Le Delta I, 2875, boul. Laurier, bureau 450, Sainte-Foy, Québec G1V 2M2 • Tél. : (418) 657-4399 - www.puq.uquebec.ca

Tiré de : Revue internationale P.M.E., vol. 14, $\mathrm{n}^{\circ} 2$, sous la direction de Pierre-André Julien. 
D’Amboise, G. et A. BAKANiBona (1990), «La planification dans les PME : une synthèse de résultats empiriques, conclusions et recommandations », Revue internationale $P M E$, vol. 3, no 2, p. 147-166.

D’Amboise, G. et J.M. Nkongolo Bakenda (1997), «Le “Grounded Theory” et ses possibilités d'utilisation à la production des connaissances sur les petites et moyennes entreprises », Document de travail, no 97-17, GRPME, Université Laval, 28 p.

DOUGHERTY, D. et T. HELLER (1994), «Illegitimacy of successful product innovation in established firms », Organization Science, vol. 5, n 2, p. 200-218.

FANKAM, J.M. (1998), «Les déterminants de la planification à l'exportation en PMI», Communication au VII ${ }^{\mathrm{e}}$ Congrès de l'AIMS, 23 p., Louvain-la-Neuve, 27-29 mai.

ForBES, A.M. (1974), «Long range planning for the smaller firm », Long Range Planning, vol. $7, \mathrm{n}^{\circ} 2$, p. 43-48.

GILMORE, F.F. (1971), «Formulating strategy in smaller companies », Harvard Business Review, mai-juin, p. 71-81.

GLASER, B. et A. STRAUSS (1967), The Discovery of Grounded Theory, Chicago, Aldine.

GOLDE, R.A. (1964), «Practical planning for small business », Harvard Business Review, septembre-octobre, p. 147-161.

Goy, H. (2000), Les réalités de la planification organisationnelle des petites et moyennes entreprises industrielles, Thèse de doctorat en sciences de gestion, Université de Grenoble II, ESA, 16 novembre, 406 p.

GREEN, L. (1982), «Planning and decision-making in the small business », Managerial Planning, vol. 31, $\mathrm{n}^{\circ} 1$, p. 27-32.

JONES, W.D. (1982), «Characteristics of planning in small firms », Journal of Small Business Management, vol. 20, $\mathrm{n}^{\circ}$ 3, p. 15-19.

KARGAR, J. (1996), «Strategic planning system characteristics and planning effectiveness in small mature firms », Mid-Atlantic Journal of Business, vol. 32, nº 1, p. 19-34.

KARGAR, J. et J.A. PARNELL (1996), «Strategic planning emphasis and planning satisfaction in small firms : an empirical investigation », Journal of Business Strategies, vol. 13, $\mathrm{n}^{\mathrm{o}} 1$, p. 42-64.

MARMUSE, C. (1979), « Pour une planification à la mesure des PME», Direction et Gestion, $\mathrm{n}^{\mathrm{o}} 4$, juillet-août.

MinTZBERG, H. (1994), Grandeur et décadence de la planification stratégique, Paris, Dunod, $456 \mathrm{p}$.

Montebello, M.H., M. SAIAS et J. GREFFEUILLE (1974), « Les P.M.I. face à la planification stratégique », Document de recherche, Stratégie et Structure, no 74-1, 22 p.

ORPEN, C. (1985), « The effects of long-range planning on small business performance: a further examination », Journal of Small Business Management, vol. 23, n 1 , p. 16-23.

RICE, G.H. (1983), «Strategic decision-making in small business », Journal of General Management, vol. 9, $\mathrm{n}^{\mathrm{0}}$ 1, p. 58-65.

Revue internationale P.M.E., vol. 14, n 2, 2001 
Rice, G.H. et R.E. Hamilton (1979), «Decision theory and the small businessman », American Journal of Small Business, vol. 4, $\mathrm{n}^{\circ}$ 1, p. 7-15.

RoBINSON, R.B. (1979), «Forecasting and small business : a study of the strategic planning process », Journal of Small Business Management, vol. 17, nº 3, p. 19-27.

RoBInSON, R.B. (1982), «The importance of "outsiders" in small firm strategic planning », Academy of Management Journal, vol. 25, no 1, p. 80-93.

ROBINSON, R.B. et al. (1984), «The relationship between stage of development and small firm planning and performance », Journal of Small Business Management, vol. 22, $n^{\circ} 2$, p. $45-52$.

RoBINSON, R.B. et al. (1986), «Planning activities related to independent retail firm performance », American Journal of Small Business, vol. 11, n 1, p. 19-26.

Robinson, R.B. et J.A. PEARCE (1983), «The impact of formalized strategic planning on financial performance in small organizations », Strategic Management Journal, vol. 4, no 3, p. 197-207.

RoBinson, R.B. et J.A. PEARCE (1984), « Research thrusts in small firm strategic planning », Academy of Management Review, vol. 9, n 1, p. 128-137.

RoBINSON, R.B., J.E. LOGAN et M.Y SALEM (1986), « Strategic versus operational planning in small retail firms », American Journal of Small Business, vol. 10, nº 3, p. 7-16.

RousseAU, B. (1992), « Recherche exploratoire sur la planification des PMI dans la périphérie de Limoges », Document de recherche, nº 97, CEREGE, 15 p.

SCARBOROUGH, N.M. et T.W. ZIMMERER (1987), « Strategic planning for the small business », Business, vol. 37, $\mathrm{n}^{\circ}$ 2, p. 11-19.

SCHWENK, C.R. et C.B. SHRADER (1993), «Effects of formal strategic planning on financial performance in small firms : a meta-analysis », Entrepreneurship : Theory and Practice, vol. 17, n 3, p. 53-64.

SEXTON, D.L. et P.M. VAN AuKEN (1982), «Prevalence of strategic planning in the small business », Journal of Small Business Management, vol. 20, $\mathrm{n}^{\circ}$ 3, p. 20-26.

SEXTON, D.L. et P.M. VAN Auken (1985), «A longitudinal study of small business strategic planning », Journal of Small Business Management, vol. 23, $\mathrm{n}^{\circ}$ 1, p. 7-15.

SEXTON, R.N. et R.D. DAHLE (1976), «Factors affecting long-range planning in the small business firm », Market Business Review, vol. 20, nº 4, p. 158-165.

SHAW, J.J., J.C. SHUMAN et G. SUSSMAN (1986), «An empirical analysis of planning methods utilized by small New England business firms », The Northeast Journal of Business \& Economics, vol. 12, nº 2, p. 54-67.

Shrader, C.B., C.L. MUlford et V.L. BlackBurN (1989), «Strategic and operational planning, uncertainty, and performance in small firms », Journal of Small Business Management, vol. 27, $\mathrm{n}^{\mathrm{0}}$ 4, p. 45-60.

SHUMAN, J.C. (1975), «Corporate planning in small companies, a survey », Long Range Planning, vol. 8, n 5, p. 81-90.

Shuman, J.C., JJ. Shaw et G. SuSSMAn (1985), « Strategic planning in smaller rapid growth companies », Long Range Planning, vol. 18, nº 6, p. 48-53.

Revue internationale P.M.E., vol. 14, n 2, 2001

(C) 2001 - Presses de l'Université du Québec

Édifice Le Delta I, 2875, boul. Laurier, bureau 450, Sainte-Foy, Québec G1V 2M2 • Tél. : (418) 657-4399 - www.puq.uquebec.ca

Tiré de : Revue internationale P.M.E., vol. $14, \mathrm{n}^{\circ} 2$, sous la direction de Pierre-André Julien. 
SteInER, G.A. (1979), Strategic Planning : What Every Manager Must Know, New York, The Free Press.

Strauss, A. (1987), Qualitative Analysis for Social Scientists, New York, Cambridge University Press.

Thomas, D.M.C. (1971), «Corporate strategy in a medium-sized company », Management Decision, vol. 9, p. 259-263.

TORRÈs, O. (1997), « Pour une approche contingente de la spécificité de la PME», Revue internationale PME, vol. 10, $\mathrm{n}^{\circ}$ 2, p. 9-43.

UnNI, V.K. (1981), «The role of strategic planning in small businesses », Long Range Planning, vol. 14, $\mathrm{n}^{\circ} 2$, p. 54-58.

VAN HoORN, T.P. (1979), «Strategic planning in small and medium-sized companies », Long Range Planning, vol. 12, $\mathrm{n}^{\circ} 2$, p. 84-91.

WAALEWIJn, P. et P. SEGAAR (1993), « Strategic management : the key to profitability in small companies », Long Range Planning, vol. 26, $\mathrm{n}^{\circ}$ 2, p. 24-30.

WHEELWRIGHT, S.C. (1971), «Strategic planning in the small business: the procedure in three corporations », Business Horizons, vol. 14, n 4, p. 51-58.

YIN, R.K. (1984), Case Study Research: Design and Methods, Beverly Hills, CA, Sage. 